\title{
From Linear to Nonlinear Responses of Thermal Pure Quantum States
}

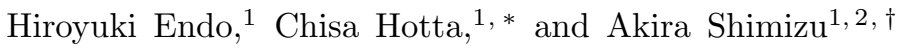 \\ ${ }^{1}$ Department of Basic Science, The University of Tokyo, 3-8-1 Komaba, Meguro, Tokyo 153-8902, Japan \\ ${ }^{2}$ Komaba Institute for Science, The University of Tokyo, 3-8-1 Komaba, Meguro, Tokyo 153-8902, Japan
}

(Dated: June 25, 2021)

\begin{abstract}
We propose a self-validating scheme to calculate the unbiased responses of quantum many-body systems to external fields of arbibraty strength at any temperature. By switching on a specified field to a thermal pure quantum state of an isolated system, and tracking its time evolution, one can observe an intrinsic thermalization process driven solely by many-body effects. The transient behavior before thermalization contains rich information on excited states, giving the linear and nonlinear response functions at all frequencies. We uncover the necessary conditions to clarify the applicability of this formalism, supported by a proper definition of the nonlinear response function. The accuracy of the protocol is guaranteed by a rigorous upper bound of error exponentially decreasing with system size, and is well implemented in the simple ferromagnetic Heisenberg chain, whose response at high fields exhibits a nonlinear band deformation. We further extract the characteristic features of excitation of the spin-1/2 kagome antiferromagnet; the wavenumber-insensitive linear responses from the possible spin liquid ground state, and the significantly broad nonlinear peaks which should be generated from numerous collisions of quasi-particles, that are beyond the perturbative description.
\end{abstract}

When studying the dynamics of quantum many-body systems, one often encounters problems to which the linear response (LR) theory does not apply [1,4]. The LR in a weak external field gives us information only on the first-order excitations. For stronger fields, a nonlinear response (NLR) arises from higher-order processes, such as multi-photon and Raman processes, which provide us with abundant information on the excitations of the system [5] 9]. Even a non-perturbative effect such as the band-gap renormalization is observed in the NLR [7, 9,

Applications of the LR includes the determination of the fluctuations at or near equilibrium [14, which is used to estimate the noises in electrical circuits [10. On the top of that, the NLR covers a wider range of phenomena including harmonic generation [5, squeezing [11, generation of entangled states [12, and quantum computation [13. Therefore, in quest for appropriate nonlinear materials, basic understanding of the NLR is demanded.

Unfortunately, it is hard to calculate or predict NLR in many-body quantum systems except for very limited cases, such as in an off-resonant field whose effects can be renormalized into the system parameters 14, 15. This situation stands in contrast to the LR, with many elaborate methods developed, such as DMRG [16 18, quantum Monte Carlo simulations [19, 20, kernel polynomial method [21, time correlation in pure quantum states 22 31], and matrix-product state [32]. Some of them were applied to NLR 24, 30, but only in a limited situation such as infinite temperature.

If the system had only a few degrees of freedom, it would require a bath in order to 'thermalize' after the quench. For such cases, numerical methods were successfully developed 33 35, where the LR and NLR would depend explicitly on the system-bath coupling. However, recent studies revealed that a many-body quantum sys- tem thermalizes even when isolated, provided that the number of conserved quantities is small 36,43 . We then expect that a series of pure states that appear during the nonequilibrium relaxation process includes abundant information on the intrinsic dynamics of the system.

In this Letter, we build a general and systematic protocol to extract the responses from the LR to the NLR regime based on the typicality approach [36, 44, 51]. Our method is applicable to general many-body quantum systems and at any temperature. We prove rigorously that the time evolution of the expectation value of any loworder polynomial of local observables agrees with that obtained from the time evolution of the Gibbs state, within an error exponentially vanishing with increasing system size. We also clarify the necessary conditions to legitimate our NLR functions. These two guarantee the fully controlled observation beyond the LR. As illustrations, we analyze the NLR to a helical magnetic field for the kagome antiferromagnet $[52,56$ as well as for the ferromagnetic Heisenberg chain.

Initial equilibrium state. - Consider a many-body quantum system with the Hamiltonian $\hat{H}$, initially $(t \leq$ 0 ) in a thermal equilibrium. Such an equilibrium state can be represented by various types of pure quantum states [36, 44 51]. Here, we choose the unnormalized canonical thermal pure quantum (cTPQ) state [50] as an initial equilibrium state given by

$$
|\beta, N\rangle=\sum_{\nu} z_{\nu} \exp [-\beta \hat{H} / 2]|\nu\rangle
$$

at inverse temperature $\beta=1 / T\left(\right.$ where $\left.k_{\mathrm{B}}=1\right)$ and system size $N$, with an arbitrary orthonormal basis of the Hilbert space $\{|\nu\rangle\}_{\nu}$, and a random complex number $z_{\nu}=\left(x_{\nu}+i y_{\nu}\right) / \sqrt{2}$ [51]. A single cTPQ state gives the free energy by $\beta F(T, N)=-\ln \langle\beta, N \mid \beta, N\rangle$, and accord- 
ingly all the thermodynamic properties within an error exponentially decreasing in $N$ 49 51 .

Response to external field. - Let us switch on an external vector field $\boldsymbol{h}$ at $t=0$,

$$
\boldsymbol{h}(\boldsymbol{x}, t)=h \boldsymbol{n}(\boldsymbol{x}) \theta(t),
$$

where $h>0, \max _{\boldsymbol{x}}|\boldsymbol{n}(\boldsymbol{x})|=1, \boldsymbol{x}$ is a spacial coordinate, and $\theta(t)$ is the step function. Suppose that $\boldsymbol{h}$ couples to the system with the interaction $\hat{H}_{\text {ext }}=-\sum_{x} \boldsymbol{h}(\boldsymbol{x}, t)$. $\hat{\boldsymbol{s}}(\boldsymbol{x})=-h \hat{B} \theta(t)$, where $\hat{\boldsymbol{s}}(\boldsymbol{x})$ is a local operator of the system, and $\hat{B}:=\sum_{\boldsymbol{x}} \boldsymbol{n}(\boldsymbol{x}) \cdot \hat{\boldsymbol{s}}(\boldsymbol{x})$.

As a response to $\boldsymbol{h}$, we focus on a certain observable $\hat{A}$, which is an additive quantity or, more generally, a low-order polynomial (such as a two-point correlation) of local observables [57. Its deviation from the initial equilibrium value is given by

$$
\Delta A(t)=\langle\hat{A}(t)\rangle_{\beta, N}-\langle\hat{A}\rangle_{\beta, N},
$$

where $\langle\bullet\rangle_{\beta, N}:=\langle\beta, N|\bullet| \beta, N\rangle /\langle\beta, N \mid \beta, N\rangle, \hat{A}(t)=$ $\hat{U}^{\dagger}(t) \hat{A} \hat{U}(t)$, and, taking $\hbar=1, \hat{U}(t)=\exp [-i(\hat{H}-h \hat{B}) t]$.

Here, similarly to what is rigorously proved for $\langle\hat{A}\rangle_{\beta, N}$ in the cTPQ state [50, we show that $\langle\hat{A}(t)\rangle_{\beta, N}$ converges in probability to the nonequilibrium value calculated from the Gibbs state $\hat{\rho}_{\beta},\langle\hat{A}(t)\rangle_{\beta, N}^{\text {ens }}=\operatorname{Tr}\left[\hat{\rho}_{\beta} \hat{A}(t)\right]$. Its deviation from the Gibbs ensemble after dropping off smaller-order terms is evaluated as,

$$
\begin{aligned}
& D[\hat{A}(t)]^{2}:=\overline{\left(\langle\hat{A}(t)\rangle_{\beta, N}-\langle\hat{A}(t)\rangle_{\beta, N}^{\mathrm{ens}}\right)^{2}} \\
& \quad \leq \frac{\left\langle(\Delta \hat{A}(t))^{2}\right\rangle_{2 \beta, N}^{\text {ens }}+\left(\langle\hat{A}(t)\rangle_{2 \beta, N}^{\text {ens }}-\langle\hat{A}(t)\rangle_{\beta, N}^{\mathrm{ens}}\right)^{2}}{\exp [2 \beta\{F(T / 2, N)-F(T, N)\}]},
\end{aligned}
$$

where $\boldsymbol{-}$ denotes average over realizations of $\left\{z_{\nu}\right\}$, and $\Delta \hat{A}(t):=\hat{A}(t)-\langle\hat{A}(t)\rangle_{2 \beta, N}^{\text {ens }}$. For every finite $\beta$, $F(T / 2, N)-F(T, N)=\Theta(N)$ [58] because the entropy $S=-\partial F / \partial T=\Theta(N)$. Hence, the denominator of the rhs of Eq. 44 is $e^{\Theta(N)}$. Now, if we consider a typical case where $\hat{A}$ is an $m$-degree polynomial of bounded local observables [59, the numerator is bounded to $\leq \Theta\left(N^{2 m}\right)$. We thus find $D[\hat{A}(t)]^{2} \leq \Theta\left(N^{2 m}\right) / e^{\Theta(N)}$, which becomes exponentially small with increasing $N$. According to a Markov type inequality, this implies that $\langle\hat{A}(t)\rangle_{\beta, N}$ converges to $\langle\hat{A}(t)\rangle_{\beta, N}^{\text {ens }}$ with probability exponentially close to one, as in the equilibrium case [49 51. Therefore, Eq. (3) gives the correct response of the system of size $N$ with exponentially small error.

Linear and nonlinear susceptibility. - The LR and NLR need to be discussed separately. When $h$ is small enough, the response extrapolates to that obtained from the LR theory [1-4. In this LR regime, the linear susceptibility (or admittance) $\chi(\omega)$, which is the Fourier transform of the LR function 1-4, does not depend on the profile of $\boldsymbol{h}$ along the time axis. Therefore, it is sufficient to consider the specific time dependent profile Eq. (2), to obtain the general form of $\chi(\omega)$ as a function of frequency $\omega$. Assuming that $\hat{A}$ is an additive observable, we obtain the following formula

$$
\chi(\omega)=\frac{\Delta A(+\infty)}{N h}-i \omega \int_{0}^{\infty} \frac{\Delta^{\prime} A(t)}{N h} e^{i \omega t} d t,
$$

where $\Delta^{\prime} A(t):=\langle\hat{A}(t)\rangle_{\beta, N}-\langle\hat{A}(+\infty)\rangle_{\beta, N}$. According to Kubo [1], $\chi(\omega)$ is explicitly given by the retarded Green function at equilibrium, which contains the information on the elementary excitations, whose nature could thus be examined by evaluating $\langle\hat{A}(t)\rangle_{\beta, N}$ for sufficiently small $h$. One can further specify the wavenumber $\boldsymbol{q}$ in $\boldsymbol{h}$, in order to obtain the $\boldsymbol{q}$-dependent susceptibility $\chi(\boldsymbol{q}, \omega)$. These points will be illustrated shortly.

At larger $h$, the correspondence with the LR theory breaks down. Still, we use Eq. (5) as the definition of the nonlinear susceptibility $\chi(\boldsymbol{q}, \omega ; h)$ with explicit $h$ dependence, because it is well-defined even in this NLR regime and is continuously connected to the linear one.

Here, we do not follow the conventional perturbative definition in nonlinear optics [5]. Our $\chi(\boldsymbol{q}, \omega ; h)$ could treat nonperturbative effects such as the nonlinear band deformation, as we see shortly.

Necessary conditions. - In actual physical systems, Eq. (5) gives correct predictions provided that $\hat{H}$ and $\hat{H}_{\text {ext }}$ are the realistic Hamiltonians [4, 60. However, in model calculations, the Hamiltonian is often too idealized, as in the case of integrable Hamiltonians obtained by neglecting small but nontrivial interactions. Usually such idealization does not affect the quality of the equilibrium properties, whereas, it often happens that they give wrong predictions about nonequilibrium properties [4, 60, 61].

To reasonably predict nonequilibrium properties of a system, the following conditions are necessary: (i) $[\hat{A}, \hat{H}-h \hat{B}] \neq 0$ because otherwise $\hat{A}$ would not respond to $\boldsymbol{h}$ at all. (ii) $[\hat{A}, \hat{H}] \neq 0$ and $[\dot{\hat{B}}, \hat{H}] \neq 0$, since otherwise the state would depend on $\boldsymbol{h}$ in the distant past, as explicitly shown in the LR regime [1-4]. (iii) In cases where $\hat{H}-h \hat{B}$ has equilibrium states [63, the equilibrium susceptibility $\chi_{\text {eq }}$ should agree with the $\omega \rightarrow 0$ limit of Eq. (5) apart from a small difference of $o(1)$ due to equilibrium fluctuations. If not, the result would be inconsistent with equilibrium statistical mechanics. Notice that the temperature rises from that of the initial state due to $\boldsymbol{h}$, and $\chi_{\text {eq }}$ should be measured at that temperature. In the LR theory, by contrast, the temperature remains the same within the order of $\Theta(h)$. Hence, condition (iii) is a generalization of that of the LR theory 61] to the NLR regime. These conditions (i)-(iii) and Eqs. (1)-(5) constitute our protocol.

Numerical method. - We employ the cTPQ state [50], $|\beta, N\rangle$, as the initial equilibrium state, and adopt the Chebyshev polynomials expansion to obtain $\hat{U}(t)$ 64. This part dominates the total numerical cost, although 

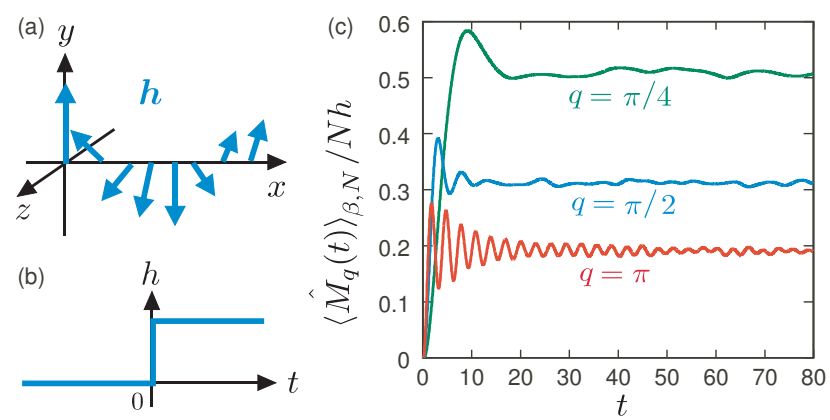

FIG. 1. (a)(b) Schematic illustration of $\boldsymbol{h}(x, t)$. (c) Time evolution of $\left\langle\hat{M}_{q}(t)\right\rangle_{\beta, N} / N h$, at $h=0.1, \beta=2$, and $N=16$.

much less costly than the full diagonalization. Throughout the time evolution, the state keeps its purity, unlike the systems coupled to baths 33 35.

Our protocol is almost self-validating in the sense that the upper bound of the error $D[\hat{A}(t)]$ in the rhs of $(4)$ is evaluated within the protocol; the denominator is calculated in a similar manner as above, and $F(T, N)$ is obtained from $\||\beta, N\rangle \|$. Notice that for small $N$ and low $T, D[\hat{A}(t)]$ can become rather large, in which case, we average over $\mathcal{M}$-independent choices of $\left\{z_{\nu}\right\}$ to reduce $D[\hat{A}(t)]$ by a factor of $1 / \sqrt{\mathcal{M}}$. [We take $\mathcal{M}=20$ and 3 in Figs. 2 and 3 respectively.]

Application to ferromagnetic Heisenberg chain.- We apply our protocol to the ferromagnetic Heisenberg chain, $\hat{H}=-\sum_{x} \hat{\boldsymbol{s}}(x) \cdot \hat{\boldsymbol{s}}(x+1)$, at $N=16$ and 24 with the periodic boundary. Here, a uniform magnetic field would not satisfy the necessary condition (ii), Instead, we set $\boldsymbol{h}=h \boldsymbol{n}(x)$ as a helical magnetic field in the $y$ - $z$ plane, i.e., $\boldsymbol{n}(x)=(0, \cos (q x), \sin (q x))$ with $q=n(2 \pi / N)$ $(n$ : integer). The spatial and time-dependent profiles of $\boldsymbol{h}$ are shown in Figs. 1 (a) and (b), respectively. Then we have $\hat{H}_{\text {ext }}=-\sum_{x} \boldsymbol{h}(x, t) \cdot \hat{\boldsymbol{s}}(x)=-h \hat{M}_{q} \theta(t)$, where $\hat{M}_{q}$ is the helical magnetization,

$$
\hat{M}_{q}:=\sum_{x}\left[\cos (q x) \hat{s}_{y}(x)+\sin (q x) \hat{s}_{z}(x)\right] .
$$

We take $\hat{M}_{q}$ also as the observable of interest, $\hat{A}$, i.e., $\hat{A}=\hat{B}=\hat{M}_{q}$. Then, $\Delta A(t)=\Delta M_{q}(t)=\left\langle\hat{M}_{q}(t)\right\rangle_{\beta, N}$ since $\left\langle\hat{M}_{q}\right\rangle_{\beta, N}=0$ in the initial equilibrium state. The above setup satisfies all the necessary conditions (i)-(iii) ((iii) has been confirmed numerically).

Figure 1(c) shows the time evolutions of $\left\langle\hat{M}_{q}(t)\right\rangle_{\beta, N} / N h$. It approaches a nearly constant value for every $q$, indicating the "thermalization" 65 67. The transient behavior of time evolution before thermalization contains rich information on the low-energy excited states, which is reflected in $\chi(q, \omega ; h)$.

Here, we focus on its imaginary part, $\operatorname{Im} \chi$, whose peak in the LR regime indicates elementary excitations. To guarantee the convergence of $\operatorname{Im} \chi$, we take a long enough

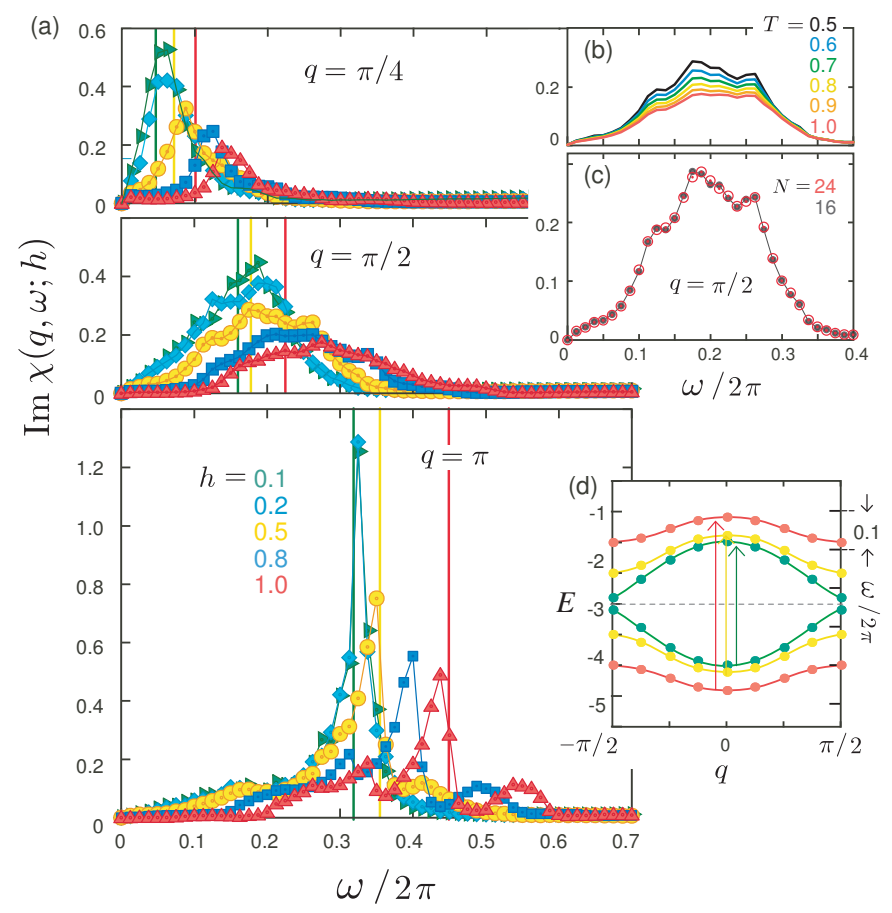

FIG. 2. Results for the ferromagnetic Heisenberg chain. (a) $\operatorname{Im} \chi(q, \omega ; h)$ at $T=0.5$ for $h=0.1-1.0, q=\pi / 4-\pi, N=16$. (b) $\operatorname{Im} \chi(q, \omega ; h)$ at $T=0.5-1.0$ for $h=0.5, q=\pi / 2, N=16$. (c) Comparison between $N=16$ and 24 at $T=0.5, h=0.5$. (d) Single-magnon dispersion in a magnetic field of $q=\pi$, whose transition energies indicated by the arrows are denoted by the vertical lines in (a) for $q=\pi$.

time-window, $t_{\text {end }}=80-160$. We further calculate the round-trip evolution $\left|\beta^{\prime}, N\right\rangle:=\hat{U}\left(-t_{\text {end }}\right) \hat{U}\left(t_{\text {end }}\right)|\beta, N\rangle$, which should equal $|\beta, N\rangle$ if the time evolution is correctly carried out. For a time step $\Delta t=1 / 50$ and the Chebyshev polynomials up to 500th order, the fidelity becomes $\left|\left\langle\beta, N \mid \beta^{\prime}, N\right\rangle\right|^{2} /\langle\beta, N \mid \beta, N\rangle\left\langle\beta^{\prime}, N \mid \beta^{\prime}, N\right\rangle=1 \pm$ $3 \times 10^{-15}$ [68. This confirms the perfect accuracy of our time evolution.

The highlight of the present protocol is the unbiased evaluation of both LR and NLR. The obtained $\operatorname{Im} \chi(q, \omega ; h)$ for $\beta=2$ are plotted in Fig. 2(a) for $q=\pi / 4, \pi / 2$ and $\pi$. At $h \gtrsim 0.1$ the peaks of the spectra show significant shift and broadening, which is a strong nonlinear effect. At lower (higher) temperature, the peaks and dips of $\chi(q, \omega ; h)$ become sharper (broader), as shown in Fig. 2(b). This happens because spins become more paramagnetic and thus less sensitive to $h$ at higher $T$. Since the finite-size effects are negligibly small (Fig. 2(c)), we concentrate on the case of $N=16$.

$L R$ regime of Heisenberg chain. - When $h \lesssim 0.1$, the response does not depend on $h ; \chi(q, \omega ; h) \rightarrow \chi(q, \omega)$ (see Supplemental Fig. S1). In this LR regime, $\chi$ agrees with the Kubo formula (we confirmed for $N=8$ ) except that the peaks and dips are broadened by $\delta \omega \sim 1 / t_{\text {end }}$ be- 
cause of the finite interval $0 \leq t \leq t_{\text {end }}$ in the Fourier transformation.

The fully polarized ground state of this model hosts a series of magnon excitations 69 73. One can construct a small subspace that is spanned by the zero-, one-, and two-magnon states 61. By directly applying the Kubo formula to this subspace, we obtain $\operatorname{Im} \chi_{\text {sub }}$ 61, which is consistent with our $\operatorname{Im} \chi$; the microscopic origin of the peaks at $h \leq 0.1$ is identified as the transitions from a few lowest one-magnon levels to the higher ones, as well as to the continuum [61. This kind of treatment works to clarify the physical origin of $\chi$, but is usually not available, e.g. in the kagome antiferromagnet we see shortly.

NLR regime of Heisenberg chain. - The spectrum at $h \gtrsim 0.1$ in Fig 2(a) shows a shift and the significant modification in its shape. Our protocol properly captures these nonlinear effects clearly beyond the scheme of the Kubo formula. Here, the term $-h \hat{M}_{q}$ cannot be treated as a small perturbation, and hence, to interpret the NLR, we diagonalize the full Hamiltonian $\hat{H}-h \hat{M}_{q}$ in the subspace we used to interpret the LR [74]. The single magnon dispersion (solid line in the Supplemental Fig. S1) is then folded by the period of $q$, and the gap opens at $k=\pi / q$, resulting in a band deformation as shown in Fig. 2(d). Then, the transition energy between the subbands at $k=0$, corresponding to the peak position, increases with $h$. Thus, this picture explains semi-quantitatively the nonlinear peak shift observed in Fig. 2(a), validating Eq. (5). However, the complete NLR spectra, beyond such a simple picture, is disclosed for the first time by our protocol.

Kagome antiferromagnet. - We now present the dynamical responses of the spin-1/2 kagome antiferromagnet that had been unreachable in any of the previous techniques. The model is considered to host a spin liquid ground state [52 54 , and a densely populated low-lying nonmagnetic excitations [55]. Figures 3(a) and 3(b) show $\operatorname{Im} \chi(q, \omega ; h)$ in the $\operatorname{LR}(h=0.05)$ [see Supplementary Fig.S2(b)] and NLR $(h=0.5)$ regimes, respectively. Here, we apply a magnetic field $\boldsymbol{h}=$ $h(0, \cos (q x), \sin (q x))$, varying along the $x$-direction with $q=n \pi / 3$ while uniform in the $y$-direction on an $N=27$ cluster [75]. Then the necessary conditions (i)-(iii) are satisfied.

The LR distinctly differs from Fig. 2 in that the three different $q$ 's all show very similar profiles (except for the peak height), i.e., a characteristic wavenumber is absent. This seems to share a common context to the featureless magnetic structure factors of the frustrated spin liquid Mott insulator [76. We also find that the first peak exists at around 0.05 , in consistency with the position of the spin gap [56, if present.

In the NLR regime, a significantly broad peak is found. In the presence of strong many-body effects, the number of collisions among correlated particles generated by the
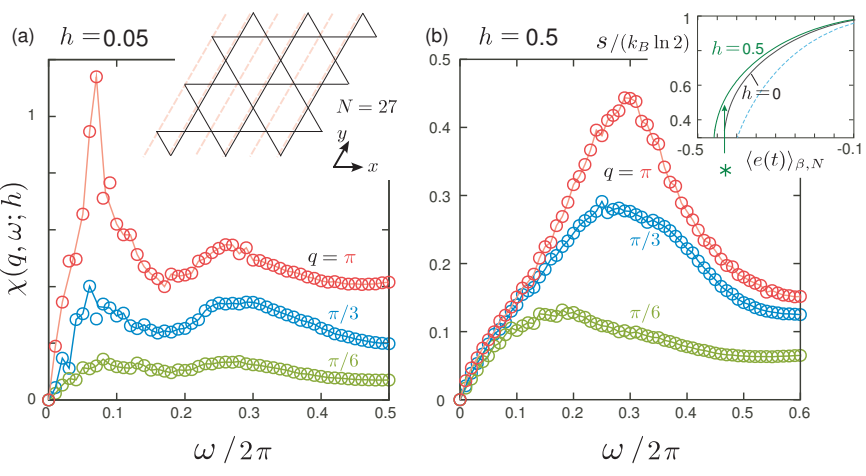

FIG. 3. Results for the spin-1/2 kagome Heisenberg antiferromagnet. $\operatorname{Im} \chi(q, \omega ; h)$ at $T=0.1$ and $N=27$ for (a) $h=0.05$, the LR regime and (b) $h=0.5$, the NLR regime. The inset shows the $N=27$ cluster. The inset of (b) is the entropy density $s /\left(k_{B} \ln 2\right)$ as a function of energy density $e$ obtained by the equilibrium microcanonical TPQ calculation [4]. Black and green solid lines are $h=0$ and $h=0.5$ $(q=\pi / 3)$ and the broken line is the case of ferromagnetic chain for comparison. The star is $\langle e(t)\rangle_{\beta, N}$ at the actual time evolution.

strong field increases rapidly, dominating the NLR. In this case, the perturbative descriptions [1, 5, break down. We expect this to happen in the present model due to large entropy density $s$; in the time evolving pure state at $q=\pi / 6$, it actually amounts to $s \sim\left(k_{B} \ln 2\right) / 2$, half of the total value (inset of Fig. 3(b)).

Concluding remarks. - If one simply replaces the observable, $\hat{A}$, of the TPQ formulation [49 51 ] with the Heisenberg operator, $\hat{A}(t)$, it easily yields wrong predictions on the LR, unless some conditions are fulfilled [2, 4, 60. Undoubtedly, this problem becomes more serious for the NLR. We provided a solution to this fundamental problem by identifying the necessary conditions (i)-(iii). It works hand in hand with the proper definition of the nonlinear susceptibility Eq. (5) which has a nonperturbative form, and the necessary conditions serve as a safeguard to avoid unphysical results. In the limit of weak fields, our susceptibility and the necessary conditions recover those of the LR theory.

On the numerical side, our protocol itself has neither restrictions on the system size (except for a limitation by available numerical resources) nor the types of models, regardless of how rapidly the entanglement grows in time evolution. So far, there had been no guarantees in both the LR and NLR for larger-scale approximate calculations. Our protocol provides a reliability check within an available system size beforehand. By computing the response function for the kagome antiferromagnet, we proved that our method is well founded even in one of the most challenging models in condensed matter.

We thank R. Hatakeyama, R. Hamazaki and K. Asano for helpful discussions, and J. Romhandyi for critical reading of the manuscript. This work is supported 
by JSPS KAKENHI Grant Numbers JP15H05700, JP26287085, JP17K05533, JP18H01173, JP17K05497, and JP17H02916.

* chisa@phys.c.u-tokyo.ac.jp

† shmz@as.c.u-tokyo.ac.jp

[1] R. Kubo, J. Phys. Soc. Jpn. 12, 570 (1957).

[2] R. Kubo, M. Toda, and N. Hashitsume, Statistical Physics II: Nonequilibrium Statistical Mechanics (Springer-Verlag, Berlin, 1985).

[3] K. Fujikura and A. Shimizu, Phys. Rev. Lett. 117, 010402 (2016).

[4] A. Shimizu and K. Fujikura, J. Stat. Mech., 024004 (2017).

[5] Y. R. Shen, The Principles of Nonlinear Optics (Wiley, New York, 1984).

[6] H. M. Gibbs, Optical Bistability: Controlling Light with Light (Academic Press, San Diego, CA, 1985).

[7] H. Haug ed. Optical Nonlinearities and Instabilities in Semiconductors (Academic Press, San Diego, CA, 1988).

[8] K. Fujii, A. Shimizu, J. Bergquist, and T. Sawada, Phys. Rev. Lett. 65, 1808 (1990).

[9] M. M. Ugeda et al., Nature Materials 13, 1091 (2014).

[10] M. J. Buckingham, Noise in Electronic Devices and Systems (John Wiley \& Sons, New York, 1983).

[11] D. F. Walls and G. J. Milburn, Quantum Optics (Springer-Verlag, Berlin, 1994).

[12] K. Edamatsu, G. Oohata, R. Shimizu, T. Itoh, Nature 431, 167 (2004).

[13] P. Adhikari, M. Hafezi, J. M. Taylor, Phys. Rev. Lett. 110, 060503 (2013).

[14] J. Inoue and A. Shimizu, J. Phys. Soc. Jpn. 68, 2534 (1999).

[15] M. Bukov, L. D'Alessio, A. Polkovnikov, Adv. Phys. 64, 139 (2015).

[16] E. Jeckelmann, Phys. Rev. B 66, 045114 (2002).

[17] K. Hallberg, Phys. Rev. B 52, R9827 (1995).

[18] T. Barthel, U. Schollwöck, and S. R. White, Phys. Rev. B 79, 245101 (2009).

[19] M. Jarrell, J. E. Gubernatis, Physics Reports 269, 133 (1996).

[20] S. Grossjohann and W. Brenig, Phys. Rev. B 79, 094409 (2009).

[21] A. Weisse, G. Wellein, A.Alvermann, and H. Fehske, Rev. Mod. Phys. 78, 275 (2006).

[22] P. de Vries and H. De Raedt, Phys. Rev. B 47, 7929 (1993).

[23] T. Iitaka and T. Ebisuzaki, Phys. Rev. Lett. 90, 047203 (2003).

[24] G. A. Alvarez, E. P. Danieli, P. R. Levstein, H. M. Pastawski, Phys. Rev. Lett. 101, 120503 (2008).

[25] J. Herbrych, R. Steinigeweg, and P. Prelovek, Phys. Rev. B 86, 1 (2012).

[26] M. Machida, T. Iitaka, and S. Miyashita, Phys. Rev. B 86, 224412 (2012).

[27] T. A. Elsayed and B. V. Fine, Phys. Rev. Lett. 110, 070404 (2013).

[28] R. Steinigeweg, J. Gemmer, W. Brenig, Phys. Rev. Lett. 112, 120601 (2014).

[29] H. Ikeuchi, H. De Raedt, S. Bertaina, and S. Miyashita
Phys. Rev. B 95, 024402 (2017).

[30] R. Steinigeweg, F. Jin, D. Schmidtke, H. De Raedt, K. Michielsen, and J. Gemmer, Phys. Rev. B 95, 035155 (2017).

[31] T. Monnai and A. Sugita, J. Phys. Soc. Jpn. 83, 094001 (2014).

[32] M. Gohlke, R. Verresen, R. Moessner, and F. Pollmann, Phys. Rev. Lett. 119, 157203 (2017).

[33] K. Molmer, Y. Castin and J. Dalibard, J. Opt. Soc. Am. 10, 524 (1993).

[34] H. Carmichael, An Open Systems Approach to Quantum Optics (Springer-Verlag, 1993). ISBN 978-0-387-56634-4.

[35] R. Dum, P. Zoller, H. Ritsch, Physical Review A. 45, 4879 (1992).

[36] J. von Neumann, Z. Phys. 57, 30 (1929).

[37] M. V. Berry, J. Phys. A 10, 2083 (1977).

[38] S. Trotzky, et al., Nat. Phys. 8, 325 (2012).

[39] J. M. Deutsch, Phys. Rev. A 43, 2046 (1991).

[40] M. Srednicki, Phys. Rev. E 50, 888 (1994).

[41] H. Tasaki, Phys. Rev. Lett. 80, 1373 (1998).

[42] M. Rigol, V. Dunjko, and M. Olshanii, Nature 452, 854 (2008).

[43] L. D'Alessio, Y. Kafri, A. Polkovnikov, and M. Rigol, Adv. Phys. 65, 239 (2016).

[44] A. Sugita, RIMS Kokyuroku (Kyoto) 1507, 147 (2006).

[45] S. Popescu, A.J. Short, and A. Winter, Nature Phys. 2, 754 (2006).

[46] S. Goldstein, J. L. Lebowitz, R. Tumulka, and N. Zanghi, Phys. Rev. Lett. 96, 050403 (2006).

[47] A. Sugita, Nonlinear Phenom. Complex Syst. 10, 192 (2007).

[48] P. Reimann, Phys. Rev. Lett. 99, 160404 (2007).

[49] S. Sugiura and A. Shimizu, Phys. Rev. Lett. 108, 240401 (2012).

[50] S. Sugiura and A. Shimizu, Phys. Rev. Lett. 111, 010401 (2013).

[51] M. Hyuga, S. Sugiura. K. Sakai and A. Shimizu, Phys. Rev. B 90, 121110(R) (2014).

[52] S. Yan, D.A. Huse, S. R. White, Science 332, 1173 (2011).

[53] S. Depenbrock, I. P. McCulloch, U. Schollwöck, Phys. Rev. Lett. 109, 067201 (2012).

[54] Y. Iqbal, F. Becca, S. Sorella, D. Poilblanc, Phys. Rev. B 87, 060405R (2013).

[55] Ch. Waldtmann , H.-U. Everts, B. Bernu, C. Lhuillier, P. Sindzingre, P. Lecheminant, and L. Pierre, Eur. Phys. J. B 2, 501 (1998)

[56] S. Nishimoto, N. Shibata, C. Hotta, Nature Comm. 4 3284 (2013).

[57] This condition on $\hat{A}$ can be relaxed to any observables whose norm is bounded by a polynomial of $N$.

[58] For a function $g(N)$, we say $g=\Theta\left(N^{k}\right)$ if $g / N^{k}$ approaches a positive constant as $N \rightarrow \infty$.

[59] Physically, the same result is expected for the case of unbounded local observables.

[60] A. Shimizu and H. Kato, Nonequilibrium Mesoscopic Conductors Driven by Reservoirs, Low-Dimensional Systems - Interactions and Transport Properties [ed. T. Brandes], Lecture Notes in Physics 54, pp.3-22 (Springer, 2000); arXiv:cond-mat/9911333

[61] Supplemental Material (which includes Ref. 62]), where we discuss condition (iii) in the LR limit, an effective model of the Heisenberg chain, and supporting results 
for the kagome antiferromagnet.

[62] X. Plat, T. Momoi, C. Hotta, Phys. Rev. B 98, 014415 (2018).

[63] Although $\boldsymbol{h}$ is static for $t \geq 0$, it does not necessarily mean that $\hat{H}-h \hat{B}$ has equilibrium states, e.g., $h \boldsymbol{n}(x)$ may be a uniform electric field (and $s(x)$ is the local charge), in which equilibrium states are impossible.

[64] H. Tal-Ezer, R. Kosloff, J. Chem. Phys. 81, 3967 (1984).

[65] A. Polkovnikov, K. Sengupta, A. Silva, M. Vengalattore, Rev. Mod. Phys. 83, 863 (2011).

[66] J. Eisert, M. Friesdorf, C. Gogolin, Nature Physics 11, 124 (2015).

[67] L. D'Alessio, Y. Kafri, A. Polkovnikov, M. Rigol, Advances in Physics, 65, 239 (2016).

[68] In practice, looser condition can still give accurate results depending on the choice of the system. For the kagome antiferromagnet, we take $\Delta t=0.2, t_{\text {end }}=100$, and the Chebyshev polynomials up to 100th order, which are enough to keep the fidelity loss as small as $\sim 10^{-10}$.

[69] F. J. Dyson, Phys. Rev. 102, 1217 (1956).

[70] F. J. Dyson, Phys. Rev. 102, 1230 (1956).

[71] M. Wortis, Phys. Rev. 132, 85 (1963).

[72] P. Longo, A. D. Greentree, K. Busch, and J. H. Cole, Phys. Lett. A 3771242 (2013).

[73] T. Fukuhara, P. Schauß, M. Endres, S. Hild, M. Cheneau, I. Bloch, and C. Gross, Nature 502, 76 (2013).

[74] H. Endo, Master Thesis, The University of Tokyo (2018).

[75] We confirmed that an $N=18$ cluster also shows a very similar broad-peak structure, with the same peak position, so that the finite size effect does not alter the result.

[76] T. Mizusaki and M. Imada, Phys. Rev. B 74, 014421 (2006). 


\title{
From Linear to Nonlinear Responses of Thermal Pure Quantum States: Supplemental Material
}

\author{
Hiroyuki Endo, ${ }^{1}$ Chisa Hotta,${ }^{1, *}$ and Akira Shimizu ${ }^{1,2, \dagger}$ \\ ${ }^{1}$ Department of Basic Science, The University of Tokyo, 3-8-1 Komaba, Meguro, Tokyo 153-8902, Japan \\ ${ }^{2}$ Komaba Institute for Science, The University of Tokyo, 3-8-1 Komaba, Meguro, Tokyo 153-8902, Japan
}

(Dated: October 24, 2018)

\section{A. Necessary condition (iii) in the limit of infinitesimal external field}

Among the three necessary conditions for the applicability of our theory, condition (iii) is the most nontrivial one. This condition is reasonable in the sense that, in the limit of $h \rightarrow 0$, it reduces to the corresponding condition for the linear response (LR) theory (i.e., the Kubo formula) [1-3]. Unfortunately, the latter condition for the LR does not seem recognized widely, despite its importance. We therefore present it briefly in this supplemental section.

We here take, as an example, the magnetic susceptibility to a uniform magnetic field. In this case, the system has an equilibrium state at $\omega=0$, with the Hamiltonian $\hat{H}-h \hat{M}$. Hence, one can check the validity of the Kubo formula by comparing its result with the results of equilibrium statistical mechanics and thermodynamics.

According to thermodynamics, the isothermal susceptibility $\chi_{T}$ takes a different value from the adiabatic susceptibility $\chi_{S}$. They are related by

$$
\chi_{S}=\chi_{T}-\frac{T}{c_{h}}\left[\left(\frac{\partial m}{\partial T}\right)_{h}\right]^{2},
$$

where $m:=M / N$, and $c_{h}$ is the specific heat at a constant magnetic field. Therefore,

$$
\chi_{T} \geq \chi_{S},
$$

and the equality holds only at the first-order phase transition (where $c_{h} \rightarrow \infty$ ) or in a nonmagnetic phase at $h=0$ (for which $m=0$ ).

Experimental results should agree with either $\chi_{T}$ or $\chi_{S}$ or another thermodynamic susceptibility, depending on the experimental conditions. Then, a question arises: Does either one of $\chi_{T}$ or $\chi_{S}$ (or another thermodynamic one) agree with $\chi_{\mathrm{K}}$ ? Here, $\chi_{\mathrm{K}}$ is the $\omega \rightarrow 0$ limit of the susceptibility obtained by the Kubo formula.

As an answer to this nontrivial question, Kubo showed in Ref. [1] that $\chi_{\mathrm{K}}=\chi_{T}$ if and only if Eq.(3.26) of [1] is satisfied. (See also Refs. [2,3].)

Condition (iii) of the present theory is a natural generalization of this requirement to the nonlinear nonequilibrium regime.

\section{B. Low energy effective model for the ferromagnetic Heisenberg chain}

In the main text, we considered the $S=1 / 2$ ferromagnetic Heisenberg chain, and calculated the response functions $\operatorname{Im} \chi_{\operatorname{sub}}(q, \omega ; h)$ based on our formula, Eq.(5).

To capture the microscopic origin of the obtained $\chi$ in Fig. 2, we consider a small subspace that is spanned by the fully polarized, one-magnon, and two-magnon states. Notice that there are low energy excited states with more magnons, while we discard them for simplicity. Figure S1 (b) shows the energy levels of $\hat{H}$ confined in this subspace. The red solid line is the one-magnon dispersion starting from the bottom of the band. This branch is fully absorbed in the magnon continuum indicated as shaded regions. At $N=16$, the energy levels $\left(E_{m}\right)$ are discrete as shown by dots, while with the increasing $N$, it apporaches the continuum, as already confirmed in the exact calculation. There is a two-magnon excitation shown in dotted lines that lies below the continuum.

Using the information on these energy levels and the corresponding eigen states, one obtains the LR within the subspace as

$$
\begin{aligned}
\operatorname{Im} \chi_{\mathrm{sub}}(q, \omega)= & \sum_{n, m}(\pi / N Z)\left(\mathrm{e}^{-\beta E_{n}}-\mathrm{e}^{-\beta E_{m}}\right) \\
& \left|\left\langle n\left|\hat{M}_{q}\right| m\right\rangle\right|^{2} \delta\left(\omega-\left(E_{m}-E_{n}\right)\right),
\end{aligned}
$$

where $Z$ is the partition function. The obtained spectra at $\beta=2$ are shown by the solid lines in Fig. S1 (a), to be compared with the full spectra $\operatorname{Im} \chi$ obtained by our protocol (symbols). One can identify the processes showing the largest contributions; the three lowest levels in Fig. S1(b) transfer to the ones separated by $q$, and the transition energies correspond to the location of the main peaks of each of the spectra. The contributions from the states not included in Fig. S1(b) are still missing in $\operatorname{Im} \chi_{\text {sub }}$, which should be the reason for their dips compared to $\operatorname{Im} \chi$. In fact the many-magnon branches goes down to the lowest energy level at $k=0$ and become all degenerate. Our $\operatorname{Im} \chi$ gives the complete profile of response. 

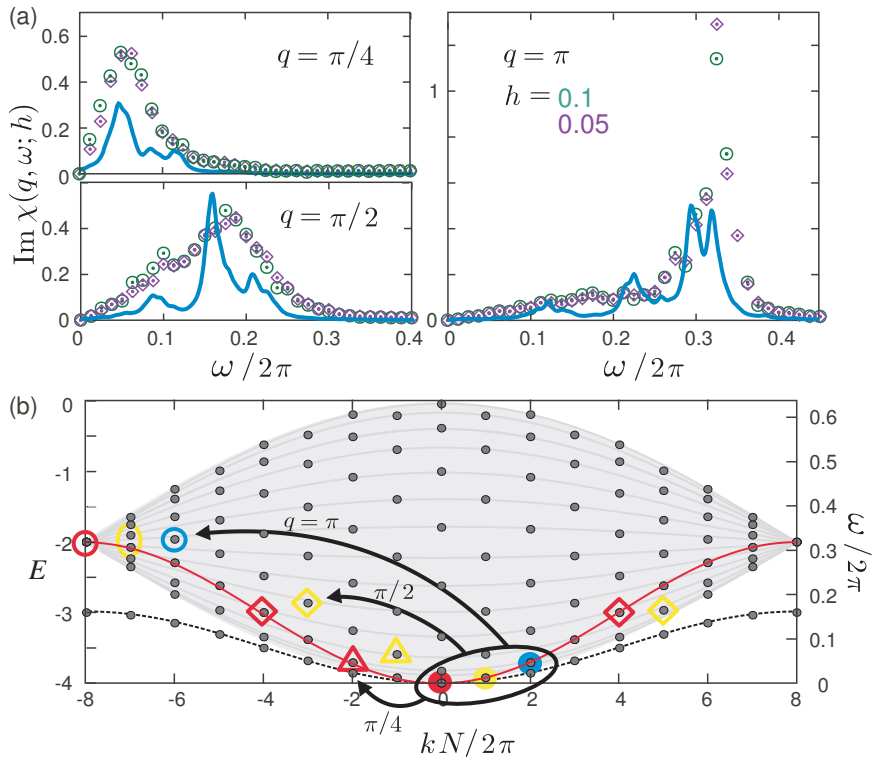

FIG. S1. (a) Comparison of $\operatorname{Im} \chi$ in Eq. (5) in the LR regime for $h=0.05$ (circles) and 0.1 (diamonds). Solid lines are $\operatorname{Im} \chi_{\text {sub }}$ defined in the text, obtained from the spectrum in panel (b), broadened by the Lorentzian of half-width $\delta \omega=1 / 2 t_{\text {end }}$. (b) Energy spectrum of the model restricted to the small subspace with up to two magnons. One magnon dispersion (red solid line), bound state of two magnons (dashed line), and the two-magnon continuum (shaded area) are shown together. The processes with large weight on the main peaks of panel (a) are shown for each $q=\pi / 4, \pi / 2, \pi$ (triangles, rectangles, open circles) starting from the three lowest energy levels (filled circles). The energy differences correspond to the peak positions.

\section{Supporting results for the kagome antiferromagnet}

The time evolution of the $S=1 / 2$ kagome antiferromagnet at $h=0.5$ in the nonlinear regime is shown in Fig. S2 (a), which gives $\chi(\boldsymbol{q}, \omega ; h)$ in Fig. $3(\mathrm{~b})$ of the main text. Each thin line gives the time evolution starting from different initial cTPQ states, and their average is shown in broken line. The sample dependence is very small where we averaged over three samples for $\chi(q, \omega ; h)$. A good convergence should be supported by the large entropy (inset of Fig. 3(b)) that is further enhanced by $h$. The asterisk symbol in inset of Fig. 3(b) indicates the energy density of the system after the quench.

We show in Fig. S2(b) the comparison of the spectrum at $h=0.02$ and 0.05 for $k_{B} T=0.1$. A good agreement shows that the system is in the linear response regime at this field strength, as in the case of ferromagnetic Heisenberg chain.

In finite cluster calculation of a two dimension, the finite size effect including the artifact due to small system size as well as the choice of the shape of the cluster may alter the result. However, in a kagome lattice antiferromagnet, quite large numbers of excited states consist of magnons that localize in space due to kinetic frustration effect [4]. In fact, as shown in Fig. S2(c), the finite size effect of the spectrum we obtained reveals as a spike, reflecting the small number of levels due to the former effect. However, the oscillation center of the spikes shows good consistency with the smooth curve at $N=27$, indicating that the feature of the spectrum is well preserved even in a system size as small as $N=18$. Thus, already at $N=27$ we find a smooth spectrum that does not alter the essential feature of the true bulk spectrum.

* chisa@phys.c.u-tokyo.ac.jp

$\dagger$ shmz@as.c.u-tokyo.ac.jp

[1] R. Kubo, J. Phys. Soc. Jpn. 12, 570 (1957).

[2] R. Kubo, M. Toda, and N. Hashitsume, Statistical Physics II: Nonequilibrium Statistical Mechanics (Springer-Verlag, Berlin, 1985).

[3] A. Shimizu and H. Kato, Nonequilibrium Mesoscopic Conductors Driven by Reservoirs, Low-Dimensional Systems - Interactions and Transport Properties [ed. T. Brandes], Lecture Notes in Physics 54, pp.3-22 (Springer, 2000); arXiv:cond-mat/9911333.

[4] X. Plat, T. Momoi, C. Hotta, Phys. Rev. B 98, 014415 (2018). 
(a) $h=0.5$

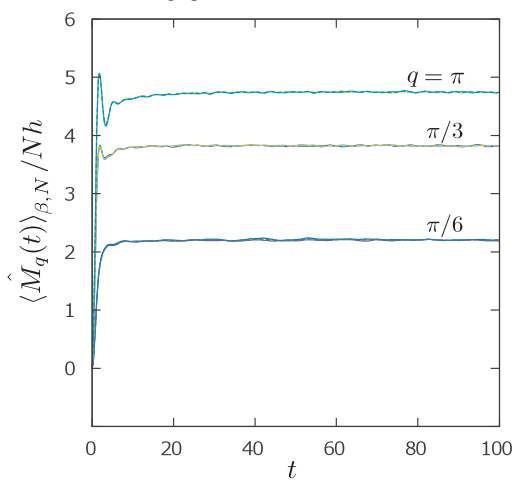

(b)

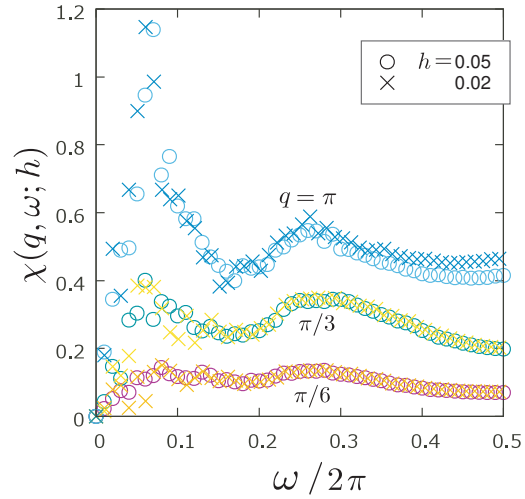

(c)

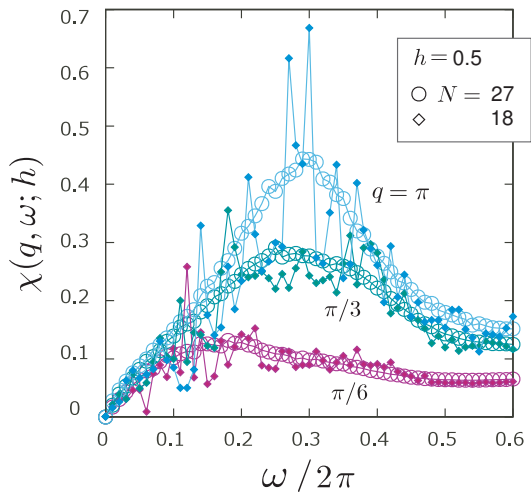

FIG. S2. Supporting results of the spin- $1 / 2$ kagome antiferromagnet at $N=27$. (a) Time evolution of $\left\langle M_{q}(t)\right\rangle$ that yields the nonlinear response (Fig. 3(b)) at $h=0.5$ and $k_{B} T=0.1$. The solid lines give the results starting from different TPQ states, and the broken lines are their averages. (b) Comparison of $\chi(q, \omega ; h)$ between $h=0.02$ and 0.05 at $k_{B} T=0.1$. The latter is the same as given in Fig. 3(a). A somewhat oscillating deviation of $h=0.02$ at small $\omega$ dissolves when taking an average of many samples (while here, we take three sample averages for all data in this figure). (c) Comparison of $\chi(q, \omega ; h)$ at $h=0.5$ between $N=27$ and 18. The spikes found in $N=18$ data is due to the small system size which is smoothed out already at $N=27$. 